Paula Luz G. Caballero, MD

Joseph E. Cachuela, MD

Department of Otorhinolaryngology

Head and Neck Surgery

Southern Philippines Medical Center, Davao City

Philippines
Correspondence: Dr. Joseph E. Cachuela

Department of Otorhinolaryngology

Head and Neck Surgery

ENT Clinic, 2nd Floor, JICA Building

Southern Philippines Medical Center

JP Laurel Avenue, Bajada, Davao City 8000

Philippines

Phone: +6382-227-2731 local 4707

Email address: j_cachuela_md@yahoo.com

The authors declared that this represents original material that is not being considered for publication or has not yet been published or accepted for publication elsewhere, in full or in part, in print or electronic media; that the manuscript has been read and approved by all the authors, that the requirements for authorship have been met by each author, and that each author believes that the manuscript represents honest work.

The authors signed disclosures that there are no financial or other (including personal) relationships, intellectual passion, political or religious beliefs, and institutional affiliations that might lead to conflict of interest.

Presented at the Philippine Society of Otolaryngology - Head and Neck Surgery Analytical Research Contest (2nd place),

November 17, 2016. Bella Ibarra, Quezon City.

Presented at Southern Philippines Medical Center 9th Annual Interdepartmental Oral Research Contest (3rd place). December 15, 2016. Southern Philippines Medical Center, Davao City.

\section{Bleeding Time Using Moringa Oleifera (Malunggay) Leaf Extract versus Saline Control in a Rabbit Epistaxis Model: A Randomized Controlled Trial}

\author{
ABSTRACT \\ Objective: To determine bleeding time using Moringa oleifera leaf extract versus saline control in \\ an experimental epistaxis model.
}

\section{Methods:}

$\begin{array}{ll}\text { Design: } & \text { Randomized controlled trial } \\ \text { Setting: } & \text { Tertiary Government Training Hospital }\end{array}$

Participants: Ten adult male New Zealand White rabbits were acclimatized for 1 week in a standard environment. One-centimeter long, full-thickness mucosal wounds in the junction of the nasal floor and anterior part of the septum were treated randomly with topical Moringa oleifera extract or colored isotonic saline control in either right or left nasal cavity, one site at a time. The duration of bleeding - time bleeding started to time bleeding stopped -- was recorded in seconds. Data was subjected to a t-test for paired samples.

Results: The mean bleeding time for wounds treated with Moringa extract was 53 seconds (range 38-70 secs), versus 159 seconds (range 100-218 secs) for controls. The bleeding time in the former was significantly shorter than in the latter $(p=.000019$, t-stat $=8.139)$, with a mean difference of 106 seconds between the two groups.

Conclusion: Moringa oleifera leaf extract was associated with significantly shorter bleeding time than saline control in this experimental epistaxis model and may be worth investigating further as a hemostatic agent for epistaxis.

Keywords: epistaxis, malunggay, Moringa oleifera extract, topical hemostatic agent

Epistaxis is one of the most common acute conditions seen in the emergency department and a common problem encountered by primary care physicians and ear, nose and throat surgeons alike. Recurrent epistaxis can be troublesome and alarming especially in the pediatric and the elderly groups. ${ }^{1}$ At least once in their lifetime, $60 \%$ of the population will experience epistaxis, $6 \%$ of which require medical attention. ${ }^{2}$ Its high frequency is associated to the nasal septum's abundant vascularity and risk for external trauma. Between 90 to $95 \%$ of epistaxis originates from Kiesselbach's plexus in the antero-inferior nasal septum. ${ }^{3}$ Traditional treatment includes nasal packing, cautery, and topical hemostatic agents. However, most of these interventions have accompanying side effects and complications such as increase in blood pressure for some of the topical hemostatic agents, infection, septal perforation and even aspiration with prolonged or 
dislodged nasal packing. Thus, investigators have continued to look for alternative methods to treat this common problem. ${ }^{3}$

Moringa oleifera, also known as malunggay, mulangay, horseradish tree, drumstick tree, or Ben oil tree, is a perennial softwood tree with low timber quality. It has long been known for its traditional medicinal as well as its industrial uses, and has been attributed with antibiotic and anti-inflammatory properties. ${ }^{4}$ Studies revealed that topical application of its extract promotes wound healing. ${ }^{5,6}$ However, its hemostatic effect on epistaxis has yet to be established. Using the keywords "Moringa," "hemostatic" and "epistaxis," we found no published study regarding the hemostatic effect of Moringa on epistaxis in a search of PubMed, the Cochrane Library, Science Direct, Philippine E-Journals, and the journals Philippine Journal of Health Research and Development, Otolaryngol Head Neck Surg, and Philipp J Otolaryngol Head Neck Surg.

In order to explore its potential hemostatic effects, the objective of this study is to determine the bleeding time using Moringa oleifera leaf extract versus saline control in an experimental epistaxis model.

\section{METHODS \\ Preparation of Moringa Extract and Control}

A modification of extract preparation yielding $13 \%$ aqueous extract was utilized. ${ }^{7}$ Fresh, green Moringa oleifera leaves procured from the local market were authenticated by a local biologist. The leaves were chopped and air-dried under shade for $72 \mathrm{hrs}$. Powdered leaves were obtained by crushing the dried leaves using a mortar and pestle. One hundred grams of powdered leaves was soaked in $500 \mathrm{ml}$ of distilled water and left standing for 48 hours at $30^{\circ} \mathrm{C}$, then filtered using Whatman No. 1 filter paper to obtain Moringa extract.

A liter of isotonic saline solution with $20 \mathrm{ml}$ of green food coloring (McCormick, McCormick Philippines Inc., Novaliches, Quezon City) made up control solution with similar appearance to the Moringa extract.

The Moringa extract and control solutions were separately put in opaque specimen bottles with $20 \mathrm{ml}$ solution per bottle. The bottles were labeled Solution A and Solution B by the principal investigator, with Solution A (Moringa extract) and Solution B (control) unknown to the blinded examiner, a licensed veterinarian. The solutions in each bottle were only used once.

\section{Animal Experiment}

Ten adult male New Zealand White rabbits with a mean weight of $1.26 \mathrm{~kg}$ (range, $1.1-1.5 \mathrm{~kg}$ ) were purchased from a certified animal distributor and acclimatized for 1 week in a standard environment. They were housed in standard cages of $1 \mathrm{~m}^{2}$ area each, in a room with a constant temperature of $22^{\circ} \mathrm{C} \pm 4^{\circ} \mathrm{C}$ and a 12-hour light/dark cycle, fed twice daily with $200 \mathrm{~g}$ generic rabbit growing pellets, and unlimited access to tap water. The study was approved by the Institutional Animal Care and Use Committee (IACUC): <IACUC Protocol Number M003>.

The experiment was conducted in the clinic of one licensed veterinarian, who performed all the procedures with one assistant. Both veterinarian and assistant were blinded.

All animals were intramuscularly sedated using $0.02 \mathrm{mg} / \mathrm{kg}$ Acepromazine (Labistress ${ }^{\circledast} 5 \mathrm{mg} / \mathrm{mL}$,Labiana Life Sciences, Barcelona, Spain) and $11 \mathrm{mg} / \mathrm{kg}$ Ketamine chloride (Ceva Ketamine $100 \mathrm{mg} / \mathrm{mL}$, Ceva Santé Animale, Sydney, Australia).

One-centimeter long full-thickness longitudinal mucosal wounds, not advancing to the cartilage, were made using Blade \#11 (Kai Medical, Kai Industries Co. Ltd., Seki City, Japan), at the junction of the nasal floor and anterior part of the septum of the right and left nasal cavity, one

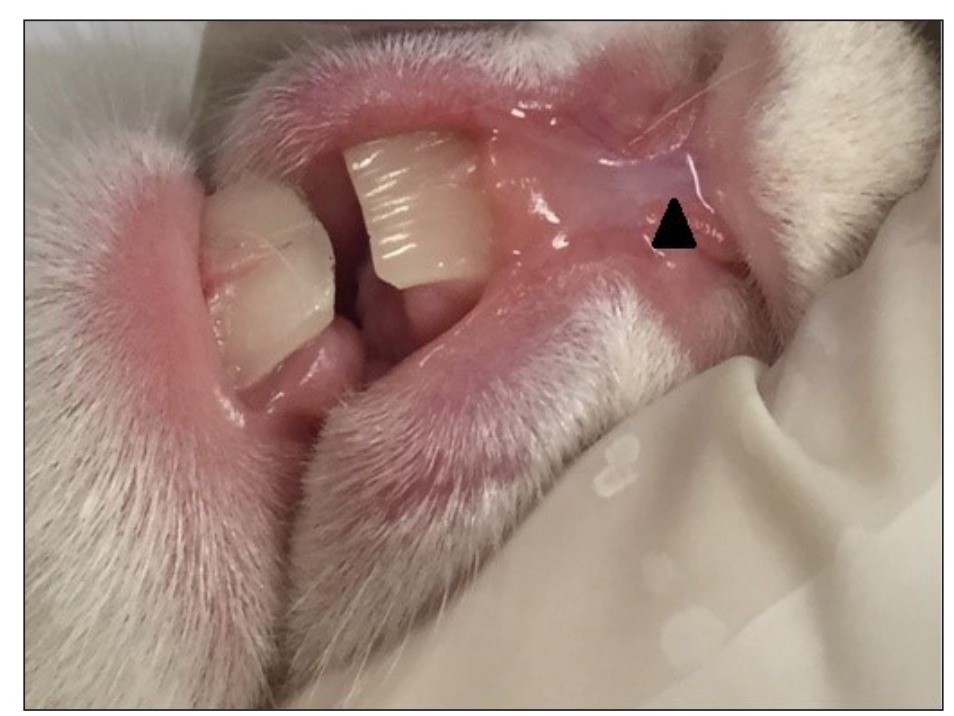

Figure 1. The area of the rabbit's nose where the incision was done ( $\mathbf{\Delta})$.

site at a time. (Figure 1) The wounds were randomized to be treated with either Solution A or B on either side.

Solutions were topically applied with cotton pledgets soaked in either solution as soon as bleeding started, and pressed gently on the wounds for 1 second, every 30 seconds (re-soaking the cotton pledget in solution for every application), until the bleeding stopped. The duration of bleeding - time bleeding started to time bleeding stopped-- was recorded in seconds by the assistant. ${ }^{3}$

Post-operative care consisted of application of topical Clotrimazole $10 \mathrm{mg}$, gentamicin sulfate $1 \mathrm{mg}$, betamethasone dipropionate $500 \mathrm{mcg}$, per gram cream (Polyderm 3, Lloyd Laboratories Inc., Malolos, Bulacan) on the wounds, right after the procedure. The rabbits were observed and monitored after 1 hour and 24 hours after conclusion of the procedure for any adverse effects or complications such as recurrence of bleeding, swelling, or mucosal irritation, and donated to a local animal habitat 3 days after the procedure. 


\section{ORIGINAL ARTICLES}

\section{Sample Size Computation}

Sample size was computed using an online sample size calculator (Select Statistical Services Ltd., Exeter, Devon, UK) to compare 2 means (http://select-statistics.co.uk/sample_size_calculation_two_means). Assuming a $95 \%$ confidence level with a power of 80 to detect a mean difference of 30 seconds with a variance of 500 seconds, 10 samples each were needed for the Moringa and control groups.

\section{Statistical Analysis}

Data was subjected to the Wilk-Shapiro test for normality (W statistic, 0.842), with computed W statistic of 0.889 (normal distribution). Data was then analyzed manually using the t-test for paired samples.

\section{RESULTS}

Ten adult male New Zealand white rabbits with ages ranging from $16-20$ weeks completed the study. None of the rabbits died or acquired any disease during the time of acclimatization. The weight of the rabbits during the study period ranged from $1.1-1.5 \mathrm{~kg}$.

The mean bleeding time for wounds administered Moringa extract was 53 seconds (range, 38 - 70 secs) compared to 159 seconds (range, $100-218$ secs) for controls. The bleeding time in the former (mean, 53.3 secs) was significantly shorter than in the latter (mean, 159.3), with a mean difference of 106 seconds between the two groups $(p=.000019$, $t$-stat $=8.139$ ). No adverse effects or complications were noted.

\section{DISCUSSION}

Mucosal healing undergoes a process that follows the stages of fibroplasia, angiogenesis, and reepithelialization. ${ }^{8}$ Topical application of Moringa oleifera extract has been shown to promote wound healing ${ }^{5,6}$ Application of the extract on wounds increases its tensile strength through rapid re-epithelialization and collagen formation. ${ }^{5}$ It also increases fibroblast proliferation. ${ }^{6}$

Moringa extract has also been known to act on the blood coagulation cascade. The presence of proteolytic activity is one of the important characteristic features of Moringa oleifera. In a study by Satish et al., both extracts from the leaves and roots of the plant showed proteolytic activity in a dose-dependent manner with the leaf extract exhibiting a significantly higher activity. The study suggested that both extracts exhibited procoagulant activity and reduced recalcification time in clot formation by activation of factors involved in the blood coagulation cascade or by precipitation of the co-factors. Both extracts also exhibited fibrinogenolytic and fibrinolytic activities. ${ }^{9}$

Whether procoagulant activity and reduced recalcification time in clot formation or fibrinogenolytic and fibrinolytic properties of Moringa extract were involved in reducing bleeding time in this study were not established, and can at best be inferred. However, the significant difference in bleeding time between treatment and controls makes a compelling argument to pursue the potential hemostatic effects of Moringa for epistaxis.

Further studies are recommended to determine the histopathologic effects of Moringa extract on nasal mucosa; its toxic dose and approximate effective dose; the effect of tonicity, $\mathrm{pH}$ and temperature settings of Moringa extract in the coagulation process; the hemostatic effect of Moringa extract versus such usual topical agents as oxymetazoline and epinephrine; sterilization techniques in the preparation of the extract; before actual clinical trials.

Our study has established that Moringa oleifera leaf extract was associated with significantly shorter bleeding time than saline control in this experimental epistaxis model, and may thus be worth investigating further as a hemostatic agent for epistaxis.

\section{ACKNOWLEDGEMENT}

The authors would like to thank Mr. Reynaldo G. Abad for authentication of the malunggay leaves, Dr. Rojim Sorrosa for sharing his inputs in the writing process, and Mr. Jesse Mari F. Santos, Mr. Arturo E. Caballero, and Mrs. Luz-Minda G. Caballero for helping the authors in processing the animal ethics approval and data gathering.

\section{REFERENCES}

1. Kotecha B, Fowler S, Harkness P, Walmsley J, Brown P, Topham J. Management of epistaxis: a national survey.Ann R Coll Surg Engl. 1996 Sep;78(5):444-446. PMID: 8881728; PMCID: PMC2502947.

2. Pope LE, Hobbs CG. Epistaxis: an update on current management. Postgrad Med J. 2005 May;81(955):309-314. DOI: 10.1136/pgmj.2004.025007. PMID: 15879044; PMCID: PMC1743269.

3. Kurtaran $\mathrm{H}$, Ark N, Ugur KS, Sert H, Ozboduroglu AA, Kosar A, et al. Effects of a topical hemostatic agent on an epistaxis model in rabbits. Curr Ther Res Clin Exp. 2010 Apr;71(2):105-110. DOI: 10.1016/j.curtheres.2010.03.003. PMID: 24683256; PMCID: PMC3967325.

4. Fahey J. Moringa oleifera: a review of the medical evidence for its nutritional, therapeutic, and prophylactic properties Part 1. Trees for Life Journal. 2005 December; 1:5. [cited 2016 January 15]. Available from: http://www.TFLJournal.org/article.php/20051201124931586.

5. Vijay L, Kumar U. Evaluation of in vivo wound healing activity of Moringa oleiferabark extracts on different wound models in rats. Pharmacologia. 2012;3(11):637-640. DOI: 10.5567/ pharmacologia.2012.637.640

6. Muhammad AA, Pauzi NAS, Arulselvan P, Abas F, Fakurazi S. In vitro wound healing potential and identification of bioactive compounds from Moringa oleiferaLam. BioMed Research International. 2013 Nov; 2013, Article ID 974580. DOI: 10.1155/2013/974580.

7. Moyo B, Masika PJ, Muchenje V. Antimicrobial activities of Moringa oleiferaLam leaf extracts African Journal of Biotechnology. 2012 Feb;11(11): 2797-2802. DOI: 10.5897/AJB10.686.

8. Wabnitz D. Factors affecting mucosal healing, reciliation, and ciliary function after Endoscopic Sinus Surgery in the sheep [dissertation]. University of Adelaide; 2005. [cited 2016 Aug 8]. Available from: http://hdl.handle.net/2440/37719.

9. Satish A, Sairam S, Ahmed F, Urooj A. Moringa oleifera Lam.: Protease activity against blood coagulation cascade. Pharmacognosy Res. 2012 Jan;4(1):44-9. DOI: 10.4103/0974-8490.91034. PMID: 22224061; PMCID: PMC3250039. 